This item was submitted to Loughborough's Research Repository by the author.

Items in Figshare are protected by copyright, with all rights reserved, unless otherwise indicated.

\title{
On a class of 2D integrable lattice equations
}

PLEASE CITE THE PUBLISHED VERSION

https://doi.org/10.1063/5.0013697

PUBLISHER

AIP Publishing

VERSION

AM (Accepted Manuscript)

PUBLISHER STATEMENT

This article may be downloaded for personal use only. Any other use requires prior permission of the author and AIP Publishing. This article appeared in Journal of Mathematical Physics, 61 (7), 073505 and may be found at https://aip.scitation.org/doi/abs/10.1063/5.0013697.

\section{LICENCE}

All Rights Reserved

\section{REPOSITORY RECORD}

Ferapontov, Evgeny, Ismagil Habibullin, Mariya Kuznetsova, and Vladimir Novikov. 2020. "On a Class of 2D Integrable Lattice Equations”. Loughborough University. https://hdl.handle.net/2134/12559481.v1. 


\title{
On a class of 2D integrable lattice equations
}

\author{
E.V. Ferapontov ${ }^{1,2}$, I.T. Habibullin ${ }^{2,3}$, M.N. Kuznetsova ${ }^{2}$, V.S. Novikov ${ }^{1}$ \\ ${ }^{1}$ Department of Mathematical Sciences \\ Loughborough University \\ Loughborough, Leicestershire LE11 3TU \\ United Kingdom \\ ${ }^{2}$ Institute of Mathematics \\ Ufa Federal Research Centre \\ Russian Academy of Sciences \\ 112 Chernyshevsky Street, Ufa 450008 \\ Russian Federation \\ ${ }^{3}$ Bashkir State University \\ 32 Validy Street, Ufa 450076 \\ Russian Federation \\ e-mails: \\ E.V.Ferapontov@lboro.ac.uk \\ habibullinismagil@gmail.com \\ mariya.n.kuznetsova@gmail.com \\ V.Novikov@lboro.ac.uk
}

\begin{abstract}
We develop a new approach to the classification of integrable equations of the form $u_{x y}=f\left(u, u_{x}, u_{y}, \triangle_{z} u \triangle_{\bar{z}} u, \triangle_{z \bar{z}} u\right)$ where $\triangle_{z}$ and $\triangle_{\bar{z}}$ are the forward/backward discrete derivatives. The following 2 -step classification procedure is proposed:

(1) First we require that the dispersionless limit of the equation is integrable, that is, its characteristic variety defines a conformal structure which is Einstein-Weyl on every solution.

(2) Secondly, to the candidate equations selected at the previous step we apply the test of Darboux integrability of reductions obtained by imposing suitable cut-off conditions.
\end{abstract}

MSC: 35L70, 35Q51, 35Q75, 53A30, 53Z05.

Keywords: 2D lattice equations, characteristic variety, Einstein-Weyl geometry, dispersionless Lax pair, Darboux integrability. 


\section{Introduction}

In this paper we develop a new approach to the classification of integrable lattice type equations in $3 \mathrm{D}$ by combining the geometric approach of [9] with the test of $[10,11]$ based on the requirement of Darboux integrability of suitably reduced equations. As an illustration we classify integrable equations of the form

$$
u_{x y}=f\left(u, u_{x}, u_{y}, \triangle_{z} u \triangle_{\bar{z}} u, \triangle_{z \bar{z}} u\right) .
$$

Familiar examples of type (1) include the Toda equation

$$
u_{x y}=e^{\triangle_{z \bar{z}} u}
$$

and the equation

$$
u_{x y}=u_{x} u_{y} \frac{\triangle_{z \bar{z}} u}{\triangle_{z} u \triangle_{\bar{z}} u}
$$

discussed in [18, 8]. We use the notation $\triangle_{z}=\frac{T_{z}-1}{\epsilon}, \triangle_{\bar{z}}=\frac{1-T_{\bar{z}}}{\epsilon}$ for the forward/backward discrete derivatives and $\triangle_{z \bar{z}}=\frac{T_{z}+T_{\bar{z}}-2}{\epsilon^{2}}$ for the symmetrised second-order discrete derivative; here $T_{z}, T_{\bar{z}}$ are the forward/backward $\epsilon$-shifts in the variable $z$. Note that dispersionless limits of the above equations (obtained as $\epsilon \rightarrow 0$ ) coincide with the Boyer-Finley equation $u_{x y}=e^{u_{z z}}$ and the equation $u_{x y}=\frac{u_{x} u_{y}}{u_{z}^{2}} u_{z z}$, respectively. Both limits belong to the class of dispersionless integrable PDEs. Further integrable examples of type (1) obtained in [10] include the equations

$$
u_{x y}=\left(u_{x}-u\right)\left(u_{y}-u\right) \frac{\triangle_{z \bar{z}} u}{\triangle_{z} u \triangle_{\bar{z}} u}+u_{x}+u_{y}-u
$$

and

$$
u_{x y}=\left(u_{x}-u^{2}-1\right)\left(u_{y}-u^{2}-1\right) \frac{\triangle_{z \bar{z}} u}{\triangle_{z} u \triangle_{\bar{z}} u}+2 u\left(u_{x}+u_{y}-u^{2}-1\right) .
$$

We emphasize that although the lattice equations (3), (4) and (5) are essentially different, their dispersionless limits are equivalent: setting $u=e^{v+x+y}$ and $u=\tan (v+x+y)$ in the dispersionless limits of (4) and (5), respectively, we obtain the dispersionless limit of equation (3) (in variable $v$ ).

The above examples suggest the following 2-step classification procedure:

(1) First we classify integrable equation of the form

$$
u_{x y}=F\left(u, u_{x}, u_{y}, u_{z}, u_{z z}\right)
$$

which can be viewed as dispersionless limits of equations (1) when $\epsilon \rightarrow 0$. This can be done by requiring that the characteristic conformal structure $[g]$ of equation (6), namely

$$
[g]=4 F_{u_{z z}} d x d y-d z^{2},
$$

is Einstein-Weyl on every solution of (6) (see section 1.1 for the necessary details). The classification results are summarised in section 2.

(2) Secondly, replacing $u_{z}$ and $u_{z z}$ in the equations obtained at the previous step by $\sqrt{\triangle_{z} u \triangle_{\bar{z}} u}$ and $\triangle_{z \bar{z}} u$, respectively, we obtain equations of type (1) which, at this stage, are our candidates for integrability. To these candidate equations we apply the test of Darboux integrability of reductions obtained by imposing suitable cut-off conditions as proposed in [10, 11]. The necessary details are provided in section 3 . 


\subsection{Dispersionless integrability and Einstein-Weyl geometry}

Recall that Einstein-Weyl geometry is a triple $(\mathbb{D},[g], \omega)$ where $\mathbb{D}$ is a symmetric connection, $[g]$ is a conformal structure and $\omega$ is a covector such that [3]:

(a) the connection $\mathbb{D}$ preserves the conformal class: $\mathbb{D}[g]=0$;

(b) the trace-free part of the symmetrized Ricci tensor of $\mathbb{D}$ vanishes.

In coordinates, this gives

$$
\mathbb{D}_{k} g_{i j}=\omega_{k} g_{i j}, \quad R_{(i j)}=\Lambda g_{i j},
$$

where $\omega=\omega_{k} d x_{k}$ is a covector, $R_{(i j)}$ is the symmetrized Ricci tensor of $\mathbb{D}$, and $\Lambda$ is some function. Note that it is sufficient to specify $[g]$ and $\omega$ only, then the first set of equations (8) uniquely defines connection $\mathbb{D}$. We recall that in three dimensions (in what follows we label coordinates $x_{1}, x_{2}, x_{3}$ as $\left.x, y, z\right)$, Einstein-Weyl equations (8) are integrable via twistor-theoretic construction [13].

It was observed in $[20,1,5,6]$ that Einstein-Weyl structures naturally arise on solutions of second-order dispersionless integrable PDEs. Furthermore, it was pointed out in [9] that the corresponding conformal structures $[g]$ are defined by the characteristic varieties of these PDEs, and the covectors $\omega$ can also be efficiently calculated. Thus, the characteristic variety of equation (6) is

$$
p_{x} p_{y}-F_{u_{z z}} p_{z}^{2}=0,
$$

and the conformal structure $[g]$ defined by the inverse matrix (to the matrix of quadratic form (9)) coincides with (7).

Example 1. The Boyer-Finley equation $u_{x y}=e^{u_{z z}}$ gives rise to the Einstein-Weyl structure [20]

$$
[g]=4 e^{u_{z z}} d x d y-d z^{2}, \quad \omega=2 u_{z z z} d z .
$$

Example 2. The equation $u_{x y}=\frac{u_{x} u_{y}}{u_{z}^{2}} u_{z z}$ gives rise to the Einstein-Weyl structure

$$
[g]=4 \frac{u_{x} u_{y}}{u_{z}^{2}} d x d y-d z^{2}, \quad \omega=2\left(\ln \frac{u_{x} u_{y}}{u_{z}^{2}}\right)_{z} d z .
$$

For second-order dispersionless PDEs, the Einstein-Weyl property of the characteristic conformal structure can be seen as an efficient and universal integrability test. It was shown in [2] that this property implies the existence of a dispersionless Lax representation in parameterdependent commuting vector fields.

\subsection{Summary of the main results}

Our main result is a complete list of integrable equations of type (1). Although the final list does not contain new integrable equations, the main purpose of the paper is to illustrate a novel approach to the classification problem: we emphasise that the general class of equations (1) could not be tackled by any of the currently existing techniques. 
Theorem 1 Modulo elementary equivalence transformations (rescalings and interchanges $x \leftrightarrow$ $y$ ), any integrable equation of type (1) is reducible to one of the following normal forms:

$$
\begin{gathered}
u_{x y}=e^{\triangle_{z \bar{z}} u} \\
u_{x y}=\frac{u_{x} u_{y}}{u}+u \triangle_{z \bar{z}} u \\
u_{x y}=u_{x} \triangle_{z \bar{z}} u \\
u_{x y}=u_{x} u_{y} \frac{\triangle_{z \bar{z}} u}{\triangle_{z} u \triangle_{\bar{z}} u} \\
u_{x y}=\left(u_{x}-u\right)\left(u_{y}-u\right) \frac{\triangle_{z \bar{z}} u}{\triangle_{z} u \triangle_{\bar{z}} u}+u_{x}+u_{y}-u \\
u_{x y}=\left(u_{x}-u^{2}-1\right)\left(u_{y}-u^{2}-1\right) \frac{\triangle_{z \bar{z}} u}{\triangle_{z} u \triangle_{\bar{z}} u}+2 u\left(u_{x}+u_{y}-u^{2}-1\right) .
\end{gathered}
$$

The proof of Theorem 1 is summarised in Section 4 .

\section{Integrable dispersionless equations $u_{x y}=F\left(u, u_{x}, u_{y}, u_{z}, u_{z z}\right)$}

The characteristic conformal structure has the form

$$
[g]=4 F_{u_{z z}} d x d y-d z^{2} .
$$

Note that we always assume that the associated covector $\omega$ depends on finite-order jets of the variable $u$. Futhermore, the jet order of $\omega$ should be by one higher than that of the conformal structure $[g]$; since $[g]$ depends on second-order derivatives of $u$, covector $\omega$ should involve no more than third-order derivatives. There are two cases to consider when we calculate the Einstein-Weyl conditions (8).

Genuinely nonlinear case $F_{u_{z z} u_{z z}} \neq 0$. One can show that in this case the covector $\omega$ has the form

$$
\omega=\left(\frac{2}{3} \frac{F_{u_{z}}}{F_{u_{z z}}}+\frac{10}{3} \frac{D_{z}\left(F_{u_{z z}}\right)}{F_{u_{z z}}}-\frac{4}{3} \frac{D_{z}\left(F_{u_{z z} u_{z z}}\right)}{F_{u_{z z} u_{z z}}}\right) d z
$$

where $D_{z}$ denotes the total $z$-derivative (note that $d x$ and $d y$ components of $\omega$ vanish identically). The Einstein-Weyl equations lead to a system of differential constraints for $F$ which result in the following two integrable dispersionless equations:

$$
\beta^{\prime}(u) u_{x y}+\beta^{\prime \prime}(u) u_{x} u_{y}=\gamma e^{\beta^{\prime}(u) u_{z z}+\beta^{\prime \prime}(u) u_{z}^{2}}+\delta
$$

and

$$
\beta^{\prime}(u) u_{x y}+\beta^{\prime \prime}(u) u_{x} u_{y}=\gamma e^{\beta^{\prime}(u) u_{z z}+\beta^{\prime \prime}(u) u_{z}^{2}+\delta \beta^{\prime}(u) u_{z}+\frac{2}{9} \delta^{2} \beta(u)} ;
$$

here $\beta(u)$ is an arbitrary function and $\gamma, \delta$ are constants (without any loss of generality one can set $\gamma=1$ ). Note that although $\beta(u)$ can be eliminated by a change of variables $\tilde{u}=\beta(u)$, this only works at the dispersionless level and is not necessarily valid for the corresponding lattice 
equations obtained by replacing $u_{z}$ and $u_{z z}$ with $\sqrt{\triangle_{z} u \triangle_{\bar{z}} u}$ and $\triangle_{z \bar{z}} u$. Thus, at this stage we will keep $\beta(u)$ arbitrary.

Quasilinear case $F_{u_{z z} u_{z z}}=0$. Let us set

$$
u_{x y}=\varphi\left(u, u_{x}, u_{y}, u_{z}\right) u_{z z}+\psi\left(u, u_{x}, u_{y}, u_{z}\right)
$$

One can show that in this case the covector $\omega$ has the form

$$
\omega=\left(2 D_{z}(\ln \varphi)+\alpha\right) d z
$$

where $D_{z}$ denotes the total $z$-derivative and $\alpha$ is some constant (as above, both $d x$ and $d y$ components of $\omega$ vanish identically). There are several subcases depending on which variables the coefficient $\varphi$ depends upon (we will assume $\varphi \neq$ const). In what follows, $\beta=\beta(u)$ is an arbitrary function of $u$ and $\alpha, \gamma, \delta$ are arbitrary constants (the constant $\alpha$ plays a distinguished role, it is the same as in $\omega$ ); we will agree that if $\alpha$ does not appear on the right-hand side of an equation below then it equals to zero in the expression for the corresponding covector $\omega$ as well.

Subcase 1: coefficient $\varphi$ depends on $u$ only, $\varphi_{u} \neq 0$. In this case the Einstein-Weyl conditions lead to the following integrable dispersionless equation:

$$
u_{x y}=\beta u_{z z}+\frac{3}{2} \alpha \beta u_{z}+\frac{\alpha^{2} \beta^{2}}{2 \beta^{\prime}}+\left(\frac{\beta^{\prime}}{\beta}-\frac{\beta^{\prime \prime}}{\beta^{\prime}}\right) u_{x} u_{y}+\frac{\beta \beta^{\prime \prime}}{\beta^{\prime}} u_{z}^{2} .
$$

Subcase 2: coefficient $\varphi$ depends on $u, u_{z}$ only, $\varphi_{u_{z}} \neq 0$. In this case we have three integrable dispersionless equations:

$$
\begin{gathered}
u_{x y}=\gamma e^{\beta u_{z}}\left(u_{z z}+\frac{\beta^{\prime}}{\beta} u_{z}^{2}\right)+\frac{\delta}{\beta}-\frac{\beta^{\prime}}{\beta} u_{x} u_{y}, \\
u_{x y}=e^{\alpha \beta+\beta^{\prime} u_{z}}\left(u_{z z}+\alpha u_{z}+\frac{\alpha}{2 \beta^{\prime}}+\frac{\beta^{\prime \prime}}{\beta^{\prime}} u_{z}^{2}\right)-\frac{\beta^{\prime \prime}}{\beta^{\prime}} u_{x} u_{y}, \\
u_{x y}=e^{\frac{1}{2} \alpha \beta+\beta^{\prime} u_{z}}\left(u_{z z}+\frac{1}{2} \alpha u_{z}+\frac{\alpha}{\beta^{\prime}}+\frac{\beta^{\prime \prime}}{\beta^{\prime}} u_{z}^{2}\right)-\frac{\beta^{\prime \prime}}{\beta^{\prime}} u_{x} u_{y} .
\end{gathered}
$$

Subcase 3: coefficient $\varphi$ depends on $u, u_{z}, u_{y}$ only, $\varphi_{u_{y}} \neq 0$. In this case we have four integrable dispersionless equations:

$$
\begin{gathered}
u_{x y}=\beta^{\prime} u_{y} u_{z z}+\left(\frac{1}{2} \alpha^{2} \beta+\frac{3}{2} \alpha \beta^{\prime} u_{z}+\beta^{\prime \prime} u_{z}^{2}\right) u_{y}-\frac{\beta^{\prime \prime}}{\beta^{\prime}} u_{x} u_{y}, \\
u_{x y}=\left(\gamma+\beta u_{y}\right)\left(u_{z z}+\frac{\delta}{\beta}+\frac{\beta^{\prime}}{\beta} u_{z}^{2}\right)-\frac{\beta^{\prime}}{\beta} u_{x} u_{y}, \\
u_{x y}=\gamma e^{\frac{1}{2} \alpha \beta+\beta^{\prime} u_{z}} u_{y}\left(\alpha+2 \beta^{\prime} u_{z z}+\alpha \beta^{\prime} u_{z}+2 \beta^{\prime \prime} u_{z}^{2}\right)-\frac{\beta^{\prime \prime}}{\beta^{\prime}} u_{x} u_{y},
\end{gathered}
$$




$$
u_{x y}=\delta e^{\beta u_{z}}\left(u_{y}+\frac{\gamma}{\beta}\right)\left(\beta u_{z z}+\beta^{\prime} u_{z}^{2}\right)-\frac{\beta^{\prime}}{\beta} u_{x} u_{y}
$$

Subcase 4: coefficient $\varphi$ depends on all four arguments $u, u_{z}, u_{y}, u_{x}$, we can assume $\varphi_{u_{x}} \neq$ $0, \varphi_{u_{y}} \neq 0$. In this case we have the following equations:

$$
\begin{gathered}
u_{x y}=\frac{2 u_{z z}+\left(4 \beta^{\prime}-\alpha\right) u_{z}+2 \beta \beta^{\prime}-\alpha \beta}{2\left(u_{z}+\beta\right)^{2}} u_{x} u_{y}, \\
u_{x y}=\frac{u_{x} u_{y}+\beta u_{x}}{\left(u_{z}+\gamma \beta\right)^{2}} u_{z z}+\frac{\left(4 \gamma \beta^{\prime}-\alpha\right) u_{z}+2 \gamma^{2} \beta \beta^{\prime}-\alpha \gamma \beta}{2\left(u_{z}+\gamma \beta\right)^{2}} u_{x} u_{y}-\frac{2 \beta^{\prime} u_{z}^{2}+\alpha \beta u_{z}+\alpha \gamma \beta^{2}}{2\left(u_{z}+\gamma \beta\right)^{2}} u_{x}, \\
u_{x y}=\frac{\left(u_{x}+\beta\right)\left(u_{y}+\delta \beta\right)}{\left(u_{z}+\gamma \beta\right)^{2}} u_{z z}+\frac{\left(4 \gamma \beta^{\prime}-\alpha\right) u_{z}+2 \gamma^{2} \beta \beta^{\prime}-\alpha \gamma \beta}{2\left(u_{z}+\gamma \beta\right)^{2}} u_{x} u_{y}-\frac{2 \beta^{\prime} u_{z}^{2}+\alpha \beta u_{z}+\alpha \gamma \beta^{2}}{2\left(u_{z}+\gamma \beta\right)^{2}}\left(u_{y}+\delta u_{x}+\delta \beta\right),
\end{gathered}
$$

note that dispersionless limits of equations (3), (4) and (5) can be obtained from equation (22) with the choice of constants $\gamma=\alpha=0, \delta=1$, and the functions $\beta(u)=0, \beta(u)=-u$ and $\beta(u)=-u^{2}-1$, respectively.

We also have the following three equations involving hyperbolic functions:

$$
\begin{gathered}
u_{x y}=\beta^{\prime} \frac{\beta^{\prime} u_{z z}+\frac{1}{2} \alpha \beta^{\prime} u_{z}+\beta^{\prime \prime} u_{z}^{2}}{\sinh ^{2}\left(\gamma+\frac{1}{2} \alpha \beta+\beta^{\prime} u_{z}\right)} u_{x} u_{y}-\frac{\beta^{\prime \prime}}{\beta^{\prime}} u_{x} u_{y}, \\
u_{x y}=\frac{\beta u_{z z}+\beta^{\prime} u_{z}^{2}}{\sinh ^{2}\left(\delta+\beta u_{z}\right)} u_{x}\left(\gamma+\beta u_{y}\right)-\frac{\beta^{\prime}}{\beta} u_{x} u_{y}, \\
u_{x y}=\frac{\left(\mu+\beta u_{x}\right)\left(\nu+\beta u_{y}\right)}{\sinh ^{2}\left(\delta+\beta u_{z}\right)} u_{z z}+\frac{\beta^{\prime}}{\beta} \frac{\mu \nu+\beta\left(\mu u_{y}+\nu u_{x}+\beta u_{x} u_{y}\right)}{\sinh ^{2}\left(\delta+\beta u_{z}\right)} u_{z}^{2}-\frac{\beta^{\prime}}{\beta} u_{x} u_{y} .
\end{gathered}
$$

The last example (25) is the most generic: it contains three constant parameters $\mu, \nu, \delta$ and an arbitrary function $\beta(u)$. This finishes the classification of integrable dispersionless equations of type (6).

\section{$3 \quad$ Integrable lattice equations}

Here we describe a classification procedure of integrable lattices based on Darboux-integrable reductions and Lie-algebraic ideas developed in [10,11]. It will be more convenient for our purposes to represent equation (1) in the equivalent lattice form,

$$
u_{n, x y}=f\left(u_{n}, u_{n, x}, u_{n, y},\left(u_{n+1}-u_{n}\right)\left(u_{n}-u_{n-1}\right), u_{n+1}-2 u_{n}+u_{n-1}\right),
$$

obtained by formally setting $\epsilon=1$ in the expressions for discrete derivatives. We will not distinguish between representations (1) and (26) in what follows. Our approach to lattice equations (26) is based on the two pivotal moments: 
- A 3D equation is integrable if it admits a large set of $2 \mathrm{D}$ reductions integrable in the sense of Darboux.

- Darboux integrability of 2D systems can be investigated by a method based on the theory of Lie-Rinehart characteristic algebras.

We emphasize that all known integrable 3D lattice equations are integrable in the sense of the following definition:

Definition 1 A lattice of the form

$$
u_{n, x y}=g\left(u_{n+1}, u_{n}, u_{n-1}, u_{n, x}, u_{n, y}\right)
$$

is said to be integrable if there exist locally analytic functions $\varphi$ and $\psi$ of two variables such that for any choice of integers $N_{1}, N_{2}$ the hyperbolic type system

$$
\begin{aligned}
& u_{N_{1}, x y}=\varphi\left(u_{N_{1}+1}, u_{N_{1}}\right), \\
& u_{n, x y}=g\left(u_{n+1}, u_{n}, u_{n-1}, u_{n, x}, u_{n, y}\right), \quad N_{1}<n<N_{2}, \\
& u_{N_{2}, x y}=\psi\left(u_{N_{2}}, u_{N_{2}-1}\right),
\end{aligned}
$$

obtained from lattice (27) by imposing cut-off conditions at $n=N_{1}$ and $n=N_{2}$, is integrable in the sense of Darboux.

Recall that Darboux integrability means that system (28) possesses $N_{2}-N_{1}+1$ nontrivial integrals in both characteristic directions. The function $\bar{u}=\left(u_{N_{1}}, \ldots, u_{N_{2}}\right)$ and its derivatives $\bar{u}_{x}, \bar{u}_{y}, \bar{u}_{x x}, \bar{u}_{y y}$, etc., are taken as dynamical variables. By definition, a function $I\left(\bar{u}, \bar{u}_{x}, \bar{u}_{x x}, \ldots\right)$ depending on a finite set of dynamical variables is an $x$-integral of system (28) if $D_{y} I=0$ where $D_{y}$ is the operator of total derivative with respect to the variable $y$. That is to say $I$ is found from the system

$$
Y I=0, \quad X_{i} I=0
$$

where

$$
X_{i}=\frac{\partial}{\partial u_{i, y}}, \quad Y=\sum_{i=N_{1}}^{N_{2}}\left(u_{i, y} \frac{\partial}{\partial u_{i}}+g_{i} \frac{\partial}{\partial u_{i, x}}+D_{x}\left(g_{i}\right) \frac{\partial}{\partial u_{i, x x}}+\cdots\right)
$$

and $g_{i}=g\left(u_{i+1}, u_{i}, u_{i-1}, u_{i, x}, u_{i, y}\right)$.

Let us consider the Lie algebra $L_{y}$ generated by the operators $Y, X_{i}$ over the ring $K$ of locally analytic functions of the dynamical variables $\bar{u}_{y}, \bar{u}_{,}, \bar{u}_{x}, \bar{u}_{x x}, \ldots$ To the standard operation $[Z, W]=Z W-W Z$ we add the following conditions: for any $Z, W \in L_{y}$ and $a, b \in K$ we require (i) $[Z, a W]=Z(a) W+a[Z, W]$ and (ii) $(a Z) b=a Z(b)$. These conditions mean that if $Z \in L_{y}$ and $a \in K$ then $a Z \in L_{y}$. The algebra $L_{y}$ defined in this way is called the Lie-Rinehart algebra [17], [16]. We will also call it the characteristic algebra in $y$-direction. In a similar way the characteristic algebra $L_{x}$ is defined.

The algebra $L_{y}$ is of finite dimension if it admits a finite basis of operators $Z_{1}, Z_{2}, \ldots, Z_{k} \in$ $L_{y}$ such that an arbitrary element $Z \in L_{y}$ can be represented as their linear combination: $Z=a_{1} Z_{1}+a_{2} Z_{2}+\cdots+a_{k} Z_{k}$; here the coefficients are functions $a_{1}, a_{2}, \ldots, a_{k} \in K$.

Our approach is based on the following key statement [22, 23]: 
Theorem 2 System (28) admits a complete set of $y$-integrals ( $x$-integrals) if and only if its characteristic algebra $L_{y}$ (respectively, $L_{x}$ ) is of finite dimension.

Corollary 1 System (28) is integrable in the sense of Darboux if both characteristic algebras $L_{x}$ and $L_{y}$ are of finite dimension.

Characteristic Lie algebras provide an effective method for classifying integrable cases of lattice (27). Here we recall the necessary results obtained by this method.

Proposition 1 (see [11]). Integrable equation of the form

$$
\begin{aligned}
u_{n, x y}=s\left(u_{n+1}, u_{n}, u_{n-1}\right) u_{n, x} u_{n, y}+\beta\left(u_{n+1},\right. & \left.u_{n}, u_{n-1}\right) u_{n, x}+ \\
& +\gamma\left(u_{n+1}, u_{n}, u_{n-1}\right) u_{n, y}+\delta\left(u_{n+1}, u_{n}, u_{n-1}\right),
\end{aligned}
$$

with the coefficient $s$ satisfying the conditions $\frac{\partial s\left(u_{n+1}, u_{n}, u_{n-1}\right)}{\partial u_{n \pm 1}} \neq 0$, can be reduced by a point transformation to one of the following forms:

$$
\begin{aligned}
& u_{n, x y}=\alpha_{n} u_{n, x} u_{n, y}, \quad \alpha_{n}=\frac{1}{u_{n}-u_{n-1}}-\frac{1}{u_{n+1}-u_{n}}=\frac{u_{n+1}-2 u_{n}+u_{n-1}}{\left(u_{n+1}-u_{n}\right)\left(u_{n}-u_{n-1}\right)}, \\
& u_{n, x y}=\alpha_{n}\left(u_{n, x}-u_{n}\right)\left(u_{n, y}-u_{n}\right)+u_{n, x}+u_{n, y}-u_{n}, \\
& u_{n, x y}=\alpha_{n}\left(u_{n, x}-u_{n}^{2}-1\right)\left(u_{n, y}-u_{n}^{2}-1\right)+2 u_{n}\left(u_{n, x}+u_{n, y}-u_{n}^{2}-1\right) .
\end{aligned}
$$

Proposition 2 (see [15]). Integrable equation of the form

$$
u_{n, x y}=g\left(u_{n+1}, u_{n}, u_{n-1}\right) u_{n, y}+\beta\left(u_{n+1}, u_{n}, u_{n-1}\right) u_{n, x}+\delta\left(u_{n+1}, u_{n}, u_{n-1}\right),
$$

where the coefficient $g$ satisfies at least one of the conditions $\frac{\partial g}{\partial u_{n+1}} \neq 0, \frac{\partial g}{\partial u_{n-1}} \neq 0$, can be reduced by a point transformation to one of the following forms:

$$
\begin{aligned}
& u_{n, x y}=\left(e^{u_{n}-u_{n-1}}-e^{u_{n+1}-u_{n}}\right) u_{n, y}, \\
& u_{n, x y}=\left(u_{n+1}-2 u_{n}+u_{n-1}\right) u_{n, y} .
\end{aligned}
$$

Equations (34) and (35) were found earlier in [18].

Proposition 3 (see [12]). A lattice of the form

$$
u_{n, x y}=g\left(u_{n+1}, u_{n}, u_{n-1}\right) \text {, }
$$

which is integrable in the sense of Definition 1, can be reduced by suitable rescalings to one of the following forms:

$$
\begin{aligned}
& u_{n, x y}=e^{\alpha u_{n}-\frac{\alpha}{2} m u_{n+1}-\frac{\alpha}{2} k u_{n-1}}+a\left(u_{n+1}, u_{n}\right)+b\left(u_{n}, u_{n-1}\right), \\
& u_{n, x y}=e^{\alpha u_{n}} u_{n+1} u_{n-1}+a\left(u_{n+1}, u_{n}\right)+b\left(u_{n}, u_{n-1}\right), \\
& u_{n, x y}=u_{n+1} u_{n-1}+a\left(u_{n+1}, u_{n}\right)+b\left(u_{n}, u_{n-1}\right), \\
& u_{n, x y}=a\left(u_{n+1}, u_{n}\right)+b\left(u_{n}, u_{n-1}\right) ;
\end{aligned}
$$

here $\alpha \neq 0$ and $m, k$ are positive integers.

The above propositions refer to rather special classes of lattice (27). The general classification problem remains out of reach at present. 


\section{Integrable lattice equations $u_{x y}=f\left(u, u_{x}, u_{y}, \triangle_{z} u \triangle_{\bar{z}} u, \triangle_{z \bar{z}} u\right)$}

In this section we prove Theorem 1 by taking dispersionless equations (10)-(25), replacing $u_{z}$ and $u_{z z}$ by $\sqrt{\triangle_{z} u \triangle_{\bar{z}} u}$ and $\triangle_{z \bar{z}} u$, respectively, and applying the methods outlined in Section 3 to the resulting candidate equations. This is done in Sections 4.1-4.4 below.

We start by proving a useful statement concerning lattices of the form

$$
u_{n, x y}=s\left(u_{n}\right) u_{n, x} u_{n, y}+r\left(u_{n+1}, u_{n}, u_{n-1}\right) .
$$

Lemma 1 If equation (41) is integrable in the sense of Definition 1, then the function

$$
\frac{\partial^{2}}{\partial u_{n+1} \partial u_{n-1}} r\left(u_{n+1}, u_{n}, u_{n-1}\right)
$$

is a quasi-polynomial in the variable $u_{n}$.

Proof. We apply the method of characteristic algebras. The following characteristic operators are associated with (41):

$$
\begin{aligned}
Y_{i} & =\frac{\partial}{\partial u_{i}}+s\left(u_{i}\right) u_{i, x} \frac{\partial}{\partial u_{i, x}}+\cdots, \\
R & =\sum_{j=N_{1}}^{N_{2}} r_{j} \frac{\partial}{\partial u_{j, x}}+\left(s\left(u_{j}\right) r_{j} u_{j, x}+r_{j, x}\right) \frac{\partial}{\partial u_{j, x x}}+\cdots,
\end{aligned}
$$

where $r_{j}=r\left(u_{j+1}, u_{j}, u_{j-1}\right)$. The commutators of the operator $D_{x}$ with the operators $Y_{i}$ and $R$ are given by the formulae

$$
\left[D_{x}, Y_{i}\right]=-s\left(u_{i}\right) u_{i, x} Y_{i}, \quad\left[D_{x}, R\right]=-\sum_{j=N_{1}}^{N_{2}} r_{j} Y_{j} .
$$

Let us assume that $N_{2}>1,-N_{1}>>1$ and concentrate on the subalgebra generated by the operators $Y_{-1}, Y_{0}, Y_{1}$ and $Z_{-1}=\left[Y_{-1}, R\right]$. By using (42) and the Jacobi identity one can prove that

$$
\left[D_{x}, Z_{-1}\right]=-s\left(u_{-1}\right) u_{-1, x} Z_{-1}-Y_{-1}\left(r_{-2}\right) Y_{-2}+\left(R\left(s\left(u_{-1}\right) u_{-1, x}\right)-Y_{-1}\left(r_{-1}\right)\right) Y_{-1}-Y_{-1}\left(r_{0}\right) Y_{0} .
$$

Let us consider the operator $Z_{-1,1}=\left[Y_{1}, Z_{-1}\right]$ for which the following commutation formula holds:

$$
\left[D_{x}, Z_{-1,1}\right]=\left(s\left(u_{1}\right) u_{1, x}-s\left(u_{-1}\right) u_{-1, x}\right) Z_{-1,1}-r_{0, u_{1} u_{-1}} Y_{0} .
$$

Let us introduce the sequence

$$
T_{0}=Z_{-1,1}, \quad T_{1}=\left[Y_{0}, T_{0}\right], \quad T_{2}=\left[Y_{0}, T_{1}\right], \ldots, T_{k+1}=\left[Y_{0}, T_{k}\right], \ldots
$$

One has the following commutation formulae:

$$
\left[D_{x}, T_{0}\right]=\left(s\left(u_{1}\right) u_{1, x}-s\left(u_{-1}\right) u_{-1, x}\right) T_{0}-\frac{\partial r_{0, u_{1} u_{-1}}}{\partial u_{0}} Y_{0}
$$




$$
\left[D_{x}, T_{k}\right]=\left(s\left(u_{1}\right) u_{1, x}-s\left(u_{-1}\right) u_{-1, x}\right) T_{k}-\frac{\partial^{k} r_{0, u_{1} u_{-1}}}{\partial u_{0}^{k}} Y_{0}
$$

Due to the fact that the characteristic algebra must have finite dimension there should exist a natural $N$ such that the operator $T_{N+1}$ is linearly expressed through the operators $T_{N}, \ldots, T_{0}$, and the operators $T_{N}, \ldots, T_{0}$ are linearly independent. Thus,

$$
T_{N+1}+\lambda_{N} T_{N}+\cdots+\lambda_{0} T_{0}=0
$$

where the coefficients $\lambda_{i}, i=0,1, \ldots N$, are functions of a finite set of dynamical variables $\bar{u}=\left(u_{N_{1}}, u_{N_{1}-1}, \ldots, u_{N_{2}}\right), \bar{u}_{x}, \bar{u}_{x x}$, etc. Commuting both sides of this equality with the operator $D_{x}$ and applying (43), (44) we obtain

$$
\begin{gathered}
\left(s\left(u_{1}\right) u_{1, x}-s\left(u_{-1}\right) u_{-1, x}\right)\left(-\lambda_{N} T_{N}-\cdots-\lambda_{1} T_{1}-\lambda_{0} T_{0}\right)-\frac{\partial^{N+1} r_{0, u_{1} u_{-1}}}{\partial u_{0}^{N+1}} Y_{0}+ \\
+D_{x}\left(\lambda_{n}\right) T_{N}+\lambda_{N}\left(\left(s\left(u_{1}\right) u_{1, x}-s\left(u_{-1}\right) u_{-1, x}\right) T_{N}-\frac{\partial^{N} r_{0, u_{1}, u_{-1}}}{\partial u_{0}^{N}} Y_{0}\right)+ \\
\cdots \\
+D_{x}\left(\lambda_{0}\right) T_{0}+\lambda_{0}\left(\left(s\left(u_{1}\right) u_{1, x}-s\left(u_{-1}\right) u_{-1, x}\right) T_{0}-\frac{\partial r_{0, u_{1} u_{-1}}}{\partial u_{0}} Y_{0}\right)=0 .
\end{gathered}
$$

Collecting the coefficients at the independent operators $T_{N}, T_{N-1}, \ldots, T_{0}$ we obtain $D_{x}\left(\lambda_{i}\right)=0$, $i=0, \ldots, N$. Therefore, $\lambda_{i}=$ const. Comparing the coefficients at $Y_{0}$ we arrive at the ODE with constant coefficients for the function $r_{0, u_{1} u_{-1}}$ :

$$
\frac{\partial^{N+1} r_{0, u_{1} u_{-1}}}{\partial u_{0}^{N+1}}+\lambda_{N} \frac{\partial^{N} r_{0, u_{1}, u_{-1}}}{\partial u_{0}^{N}}+\cdots+\lambda_{0} \frac{\partial r_{0, u_{1} u_{-1}}}{\partial u_{0}}=0 .
$$

Each solution $r_{0, u_{1} u_{-1}}$ of this equation is a quasi-polynomial in $u_{0}$. Lemma 1 is proved.

Below, when studying equations (10)-(25), we assume that the function $\beta$ is analytic in a domain $D \subset \mathbb{C}$.

\subsection{Genuinely nonlinear case}

Here we show that the only integrable equation of the form (1) resulting from dispersionless equations (10), (11) is

$$
u_{n, x y}=e^{\triangle_{z \bar{z}} u} .
$$

Replacing $u_{z}$ and $u_{z z}$ by $\sqrt{\triangle_{z} u \triangle_{\bar{z}} u}$ and $\triangle_{z \bar{z}} u$, respectively, we obtain equations of the following form:

$$
\begin{gathered}
\beta^{\prime}\left(u_{n}\right) u_{n, x y}+\beta^{\prime \prime}\left(u_{n}\right) u_{n, x} u_{n, y}=e^{\beta^{\prime}\left(u_{n}\right)\left(u_{n+1}-2 u_{n}+u_{n-1}\right)+\beta^{\prime \prime}\left(u_{n}\right)\left(u_{n+1}-u_{n}\right)\left(u_{n}-u_{n-1}\right)}+\delta, \\
\beta^{\prime}\left(u_{n}\right) u_{n, x y}+\beta^{\prime \prime}\left(u_{n}\right) u_{n, x} u_{n, y}=e^{\beta^{\prime}\left(u_{n}\right)\left(u_{n+1}-2 u_{n}+u_{n-1}\right)+\beta^{\prime \prime}\left(u_{n}\right)\left(u_{n+1}-u_{n}\right)\left(u_{n}-u_{n-1}\right)} \times \\
\times e^{\delta \beta^{\prime}\left(u_{n}\right) \sqrt{\left(u_{n+1}-u_{n}\right)\left(u_{n}-u_{n-1}\right)}+\frac{2}{9} \delta^{2} \beta\left(u_{n}\right)} .
\end{gathered}
$$


Note that these equations belong to the subclass (41). Thus we can apply Lemma 1. A simple analysis shows that the function $r_{n, u_{n+1} u_{n-1}}$ corresponding to the equation (46) is a quasipolynomial in $u_{n}$ only if $\delta=0$. Consequently (46) is a particular case of (45) and we can focus on the latter. After the change of variables $v_{n}=\beta\left(u_{n}\right) \leftrightarrow u_{n}=\varphi\left(v_{n}\right)$ equation (45) takes the form

$$
v_{n, x y}=e^{\beta^{\prime}\left(\varphi\left(v_{n}\right)\right)\left(\varphi\left(v_{n+1}\right)-2 \varphi\left(v_{n}\right)+\varphi\left(v_{n-1}\right)\right)+\beta^{\prime \prime}\left(\varphi\left(v_{n}\right)\right)\left(\varphi\left(v_{n+1}\right)-\varphi\left(v_{n}\right)\right)\left(\varphi\left(v_{n}\right)-\varphi\left(v_{n-1}\right)\right)}+\delta,
$$

where the function $\varphi$ is the inverse of $\beta$. Let us denote by $\bar{f}\left(v_{n+1}, v_{n}, v_{n-1}\right)$ the right-hand side of this equation and evaluate the second-order derivative of $\bar{f}$ :

$$
\begin{aligned}
\bar{f}_{v_{n+1}, v_{n-1}}=( & \left.\bar{f}\left(v_{n+1}, v_{n}, v_{n-1}\right)-\delta\right) \varphi^{\prime}\left(v_{n+1}\right) \varphi^{\prime}\left(v_{n-1}\right)\left(-\beta^{\prime \prime}\left(u_{n}\right)+\right. \\
& \left.+\left(\beta^{\prime}\left(u_{n}\right)+\beta^{\prime \prime}\left(u_{n}\right)\left(\varphi\left(v_{n}\right)-\varphi\left(v_{n-1}\right)\right)\right)\left(\beta^{\prime}\left(u_{n}\right)-\beta^{\prime \prime}\left(u_{n}\right)\left(\varphi\left(v_{n+1}\right)-\varphi\left(v_{n}\right)\right)\right)\right) .
\end{aligned}
$$

Due to Proposition 3 , in the integrable case the function $\bar{f}\left(v_{n+1}, v_{n}, v_{n-1}\right)$ is a quasipolynomial in all three variables such that

$$
\bar{f}_{v_{n+1}, v_{n-1}}=C e^{\alpha v_{n}-\frac{\alpha m}{2} v_{n+1}-\frac{\alpha k}{2} v_{n-1}},
$$

where $C, \alpha$ are arbitrary constants and $m, k$ are nonnegative integers related to each other in the following way: if $m=0(k=0)$ then necessarily $k=0(m=0)$. Since equation (47) is complicated we first impose a reduction $v_{n+1}=v_{n}=v_{n-1}=v$ that obviously implies $\bar{f}-\delta=1$. Now by comparing two representations for $\bar{f}_{v_{n+1} v_{n-1}}$ under the reduction we get (due to the equation $\left.\beta^{\prime}(u)=\frac{1}{\varphi^{\prime}(v)}\right)$

$$
\beta^{\prime \prime}=\beta^{\prime 2}\left(C e^{\alpha_{1} \beta}-1\right),
$$

where $\alpha_{1}=\alpha\left(1-\frac{m}{2}-\frac{k}{2}\right)$. In terms of the function $\varphi(v)$ the equation takes the form

$$
\frac{\varphi^{\prime \prime}}{\varphi^{\prime}}=C e^{\alpha_{1} v}-1
$$

For $\alpha_{1}=0, C \neq 1$ we get

$$
\text { a) } \varphi=e^{(C-1) v} \frac{c_{2}}{C-1}+C_{3} .
$$

For $\alpha_{1}=0, C=1$ we find $\varphi^{\prime \prime}=0$ so that

$$
\text { b) } \varphi=c_{1} v+c_{2} \text {. }
$$

For $\alpha_{1} \neq 0, C \neq 0$ the solution of $(48)$ is

$$
\text { c) } \varphi=\int e^{-v} c_{2} e^{\frac{C}{\alpha_{1}} e^{\alpha_{1} v}} \mathrm{~d} v \text {. }
$$

For $\alpha_{1} \neq 0, C=0$ we have

$$
\text { d) } \varphi=c_{2}-e^{c_{1}-v} \text {. }
$$


According to Proposition $3, \bar{f}$ is a quasi-polynomial in the variables $v_{n+1}, v_{n}, v_{n-1}$. Let us take $v_{n}=v_{n-1}$ and note that the function

$$
\bar{f}\left(v_{n+1}, v_{n}, v_{n}\right)=\exp \left(\beta^{\prime}\left(u_{n}\right) \varphi\left(v_{n+1}\right)-\beta^{\prime}\left(u_{n}\right) \varphi\left(v_{n}\right)\right)
$$

has to be a quasi-polynomial in $v_{n+1}$. This is only possible in the case $b$ ). Then equation (45) takes the form

$$
\frac{1}{c_{1}} u_{n, x y}=e^{\frac{1}{c_{1}}\left(u_{n+1}-2 u_{n}+u_{n-1}\right)}+\delta
$$

which can be reduced to the first case of Theorem 1 by the change of variables $u_{n} \rightarrow c_{1} u_{n}+c_{1} \delta x y$.

\subsection{Subcases 1 and 2}

Below we prove that dispersionless equations (12)-(15) give rise to the following integrable equation of the form (1):

$$
u_{x y}=\frac{u_{x} u_{y}}{u}+u \triangle_{z \bar{z}} u
$$

Replacing $u_{z}$ and $u_{z z}$ by $\sqrt{\triangle_{z} u \triangle_{\bar{z}} u}$ and $\triangle_{z \bar{z}} u$, respectively, we obtain equations of the following form:

$$
\begin{aligned}
& u_{n, x y}=\left(\frac{\beta^{\prime}\left(u_{n}\right)}{\beta\left(u_{n}\right)}-\frac{\beta^{\prime \prime}\left(u_{n}\right)}{\beta^{\prime}\left(u_{n}\right)}\right) u_{n, x} u_{n, y}+\frac{\beta\left(u_{n}\right) \beta^{\prime \prime}\left(u_{n}\right)}{\beta^{\prime}\left(u_{n}\right)}\left(u_{n+1}-u_{n}\right)\left(u_{n}-u_{n-1}\right)+ \\
&+\beta\left(u_{n}\right)\left(u_{n+1}-2 u_{n}+u_{n-1}\right)+\frac{3}{2} \alpha \beta\left(u_{n}\right) \sqrt{\left(u_{n+1}-u_{n}\right)\left(u_{n}-u_{n-1}\right)}+\alpha^{2} \frac{\beta^{2}\left(u_{n}\right)}{2 \beta^{\prime}\left(u_{n}\right)}, \\
& u_{n, x y}= \gamma e^{\beta\left(u_{n}\right) \sqrt{\left(u_{n+1}-u_{n}\right)\left(u_{n}-u_{n-1}\right)} \times}+\frac{\delta}{\beta\left(u_{n}\right)}-\frac{\beta^{\prime}\left(u_{n}\right)}{\beta\left(u_{n}\right)} u_{n, x} u_{n, y}, \\
& \times\left(u_{n+1}-2 u_{n}+u_{n-1}+\frac{\beta^{\prime}\left(u_{n}\right)}{\beta\left(u_{n}\right)}\left(u_{n+1}-u_{n}\right)\left(u_{n}-u_{n-1}\right)\right)+ \\
& u_{n, x y}= e^{\alpha \beta\left(u_{n}\right)+\beta^{\prime}\left(u_{n}\right) \sqrt{\left(u_{n+1}-u_{n}\right)\left(u_{n}-u_{n-1}\right)} \times}-\frac{\beta^{\prime \prime}\left(u_{n}\right)}{\beta^{\prime}\left(u_{n}\right)} u_{n, x} u_{n, y}, \\
& \times\left(u_{n+1}-2 u_{n}+u_{n-1}+\frac{\alpha}{2 \beta^{\prime}\left(u_{n}\right)}+\frac{\beta^{\prime \prime}\left(u_{n}\right)}{\beta^{\prime}\left(u_{n}\right)}\left(u_{n+1}-u_{n}\right)\left(u_{n}-u_{n-1}\right)\right)- \\
& \quad \times\left(u_{n+1}-2 u_{n}+u_{n-1}+\frac{1}{2} \alpha \sqrt{\left(u_{n+1}-u_{n}\right)\left(u_{n}-u_{n-1}\right)}+\right. \\
&\left.\quad+\frac{\alpha}{\beta^{\prime}\left(u_{n}\right)}+\frac{\beta^{\prime \prime}\left(u_{n}\right)}{\beta^{\prime}\left(u_{n}\right)}\left(u_{n+1}-u_{n}\right)\left(u_{n}-u_{n-1}\right)\right)-\frac{\beta^{\prime \prime}\left(u_{n}\right)}{\beta^{\prime}\left(u_{n}\right)} u_{n, x} u_{n, y} .
\end{aligned}
$$


Note that these equations belong to the subclass (41). Thus Lemma 1 applies. A simple analysis shows that the function $r_{n, u_{n+1} u_{n-1}}$ corresponding to equations (51), (52) is actually never a quasi-polynomial in $u_{n}$, since $\beta^{\prime}$ does not vanish. Similarly we show that for the cases (49), (50) one should have $\alpha=0, \gamma=0$ (note that equation (50) with $\gamma=0$ does not contain the second-order discrete derivative, and therefore has a degenerate dispersionless limit). Therefore, equations (51), (52) are certainly non-integrable, while integrable cases of the equations (49), (50) reduce to

$$
\begin{aligned}
u_{n, x y} & =\left(\frac{\beta^{\prime}\left(u_{n}\right)}{\beta\left(u_{n}\right)}-\frac{\beta^{\prime \prime}\left(u_{n}\right)}{\beta^{\prime}\left(u_{n}\right)}\right) u_{n, x} u_{n, y}+ \\
& +\frac{\beta\left(u_{n}\right) \beta^{\prime \prime}\left(u_{n}\right)}{\beta^{\prime}\left(u_{n}\right)}\left(u_{n+1}-u_{n}\right)\left(u_{n}-u_{n-1}\right)+\beta\left(u_{n}\right)\left(u_{n+1}-2 u_{n}+u_{n-1}\right) .
\end{aligned}
$$

By a point transformation of the form $u_{n}=\varphi\left(v_{n}\right)$ we can eliminate in (53) the term containing the product $u_{n, x} u_{n, y}$. To this aim we choose $\varphi$ such that $\beta\left(\varphi\left(v_{n}\right)\right)=e^{v_{n}}$. Thus,

$$
\beta\left(u_{n}\right)=e^{v_{n}}, \quad \beta^{\prime}\left(u_{n}\right)=\frac{1}{\varphi^{\prime}\left(v_{n}\right)} e^{v_{n}}, \quad \beta^{\prime \prime}\left(u_{n}\right)=\frac{e^{v_{n}}}{\varphi^{\prime 2}\left(v_{n}\right)}\left(1-\frac{\varphi^{\prime \prime}\left(v_{n}\right)}{\varphi^{\prime}\left(v_{n}\right)}\right) .
$$

After that equation (53) takes the form

$$
\begin{aligned}
v_{n, x y}=\frac{e^{v_{n}}}{\varphi^{\prime}\left(v_{n}\right)}\left(\varphi\left(v_{n+1}\right)\right. & \left.-2 \varphi\left(v_{n}\right)+\varphi\left(v_{n-1}\right)\right)+ \\
& +\frac{e^{v_{n}}}{\varphi^{\prime 2}\left(v_{n}\right)}\left(1-\frac{\varphi^{\prime \prime}\left(v_{n}\right)}{\varphi^{\prime}\left(v_{n}\right)}\right)\left(\varphi\left(v_{n+1}\right)-\varphi\left(v_{n}\right)\right)\left(\varphi\left(v_{n}\right)-\varphi\left(v_{n-1}\right)\right) .
\end{aligned}
$$

We see that this equation belongs to the subclass (36). To study (54) we apply Proposition 3. According to (37)-(40) equation (54) can take one of the following forms:

$$
\begin{aligned}
v_{n, x y} & =e^{v_{n+1}}-2 e^{v_{n}}+e^{v_{n-1}}, \\
v_{n, x y} & =e^{v_{n}}\left(v_{n}^{2}+v_{n+1} v_{n-1}-v_{n+1} v_{n}-v_{n} v_{n-1}+2 v_{n}-v_{n+1}-v_{n-1}\right), \\
v_{n, x y} & =-(m-2) e^{-v_{n}+\frac{m}{2} v_{n+1}+\frac{m}{2} v_{n-1}}+2(m-1) e^{\left(-1+\frac{m}{2}\right) v_{n}+\frac{m}{2} v_{n+1}}+ \\
& +2(m-1) e^{\left(-1+\frac{m}{2}\right) v_{n}+\frac{m}{2} v_{n-1}}+(2-3 m) e^{(-1+m) v_{n}},
\end{aligned}
$$

where $m$ is a positive integer. Equation (55) and equation (57) with $m=1$ are known to be integrable. Equation (57) with $m=1$ corresponds to the first case of Theorem 1 (by the change of variables $v_{n} \rightarrow 2 v_{n}, x \rightarrow 2 x$ ). Equation (55) corresponds to the second case of Theorem 1 . The case $m=2$ leads to a trivial degenerate equation, hence in our further study we suppose $(m-2)(m-1) \neq 0$.

Now let us prove that equation (56) is not integrable. To this aim we investigate the characteristic algebra of this equation generated by the operators

$$
X_{j}=\frac{\partial}{\partial v_{j}}, \quad Z=\sum_{i=N_{1}}^{N_{2}}\left(f_{j} \frac{\partial}{\partial v_{j, x}}+D_{x}\left(f_{j}\right) \frac{\partial}{\partial v_{j, x x}}+\cdots\right),
$$


which satisfy the relations

$$
\left[D_{x}, X_{j}\right]=0, \quad\left[D_{x}, Z\right]=-\sum_{j=N_{1}}^{N_{2}} f_{j} X_{j}
$$

where $f_{j}=f\left(v_{j+1}, v_{j}, v_{j-1}\right)$ is the right-hand side of equation (56) represented as $v_{j, x y}=f_{j}$. We will need the following useful statement [19, 22]:

Lemma 2 If a vector field of the form

$$
Z=\sum_{i=0}^{N} z_{1, i} \frac{\partial}{\partial v_{i, x}}+z_{2, i} \frac{\partial}{\partial v_{i, x x}}+\cdots
$$

solves the equation $\left[D_{x}, Z\right]=0$, then $Z=0$.

Let us introduce the operators

$$
Z_{0}=\left[X_{0}, Z\right], \quad W_{0}=\left[X_{1}, Z_{0}\right], \quad W_{1}=\left[X_{0}, W_{0}\right], \quad W_{2}=\left[X_{0}, W_{1}\right], \quad W_{3}=\left[X_{0}, W_{2}\right] .
$$

Using the Jacobi identity and (58) one can find that

$$
\begin{gathered}
{\left[D_{x}, Z_{0}\right]=-X_{0}\left(f_{-1}\right) X_{-1}-X_{0}\left(f_{0}\right) X_{0}-X_{0}\left(f_{1}\right) X_{1}=-e^{v_{-1}}\left(v_{-2}-v_{-1}-1\right) X_{-1}-} \\
-e^{v_{0}}\left(v_{0}^{2}+v_{1} v_{-1}-v_{1} v_{0}-v_{0} v_{-1}+4 v_{0}-2 v_{1}-2 v_{-1}+2\right) X_{0}- \\
-e^{v_{1}}\left(v_{2}-v_{1}-1\right) X_{1}, \\
{\left[D_{x}, W_{0}\right]=-e^{v_{0}}\left(v_{-1}-v_{0}-2\right) X_{0}-e^{v_{1}}\left(v_{2}-v_{1}-2\right) X_{1},} \\
{\left[D_{x}, W_{k}\right]=-e^{v_{0}}\left(v_{-1}-v_{0}-(k+2)\right) X_{0}, \quad k=1,2,3 .}
\end{gathered}
$$

The next step is to introduce the operators $P=W_{3}-W_{2}, Q=W_{2}+4 P$ for which the following formulae hold:

$$
\left[D_{x}, P\right]=-e^{v_{0}} X_{0}, \quad\left[D_{x}, Q\right]=-e^{v_{0}}\left(v_{-1}-v_{0}\right) X_{0} .
$$

The function $g=-e^{v_{0}}\left(v_{-1}-v_{0}\right)$ satisfying the relation $\left[D_{x}, Q\right]=g X_{0}$ is annihilated by the operator $\Lambda\left(X_{0}\right)=\left(X_{0}-1\right)^{2}$. In other words, the polynomial $\Lambda(\lambda)$ corresponding to the operator $Q$ has a multiple root. Let us prove that in this case the characteristic subalgebra generated by the operators $P, Q$ is infinite-dimensional. To this aim we construct a sequence of operators via the following formulae:

$$
P, \quad Q, \quad K_{1}=[P, Q], \quad K_{2}=\left[P, K_{1}\right], \ldots, \quad K_{m+1}=\left[P, K_{m}\right], \ldots
$$

In order to calculate the value of the expression $\left[D_{x}, K_{1}\right]$ we need to know $\left[X_{0}, P\right],\left[X_{0}, Q\right]$ :

$$
\begin{aligned}
& {\left[D_{x},\left[X_{0}, P\right]\right]=\left[X_{0},\left[D_{x}, P\right]\right]-\left[P,\left[D_{x}, X_{0}\right]\right]=\left[X_{0},-e^{v_{0}} X_{0}\right]=-e^{v_{0}} X_{0}=\left[D_{x}, P\right],} \\
& {\left[D_{x},\left[X_{0}, Q\right]\right]=\left[X_{0},\left[D_{x}, Q\right]\right]-\left[Q,\left[D_{x}, X_{0}\right]\right]=\left[X_{0},\left[D_{x}, Q\right]\right]=} \\
& =\left[X_{0}, e^{v_{0}}\left(v_{0}-v_{-1}\right) X_{0}\right]=e^{v_{0}}\left(v_{0}-v_{-1}+1\right) X_{0}=\left[D_{x}, Q\right]-\left[D_{x}, P\right]=\left[D_{x}, Q-P\right] .
\end{aligned}
$$


Using Lemma 2 we conclude that $\left[X_{0}, P\right]=P,\left[X_{0}, Q\right]=Q-P$. Now one can find

$$
\begin{aligned}
& {\left[D_{x}, K_{1}\right]=\left[D_{x},[P, Q]\right]=\left[P,\left[D_{x}, Q\right]\right]-\left[Q,\left[D_{x}, P\right]\right]=} \\
& =\left[P, e^{v_{0}}\left(v_{0}-v_{-1}\right) X_{0}\right]-\left[Q,-e^{v_{0}} X_{0}\right]=-e^{v_{0}}\left(v_{0}-v_{-1}-1\right) P-e^{v_{0}} Q .
\end{aligned}
$$

Similarly we find

$$
\begin{aligned}
& {\left[X_{0}, K_{1}\right]=2 K_{1}, \quad\left[D_{x}, K_{2}\right]=-3 e^{v_{0}} K_{1},} \\
& {\left[X_{0}, K_{2}\right]=3 K_{2}, \quad\left[D_{x}, K_{3}\right]=-6 e^{v_{0}} K_{2} .}
\end{aligned}
$$

It can be proved by induction that the following formula holds:

$$
\left[D_{x}, K_{m}\right]=-\frac{m(m+1)}{2} e^{v_{0}} K_{m-1}, \quad m \geq 2 .
$$

Since the characteristic algebra generated by the operators $P, Q$ must be finite-dimensional, one of the two cases must hold:

1) there is an integer $M$ such that $K_{M+1}$ is expressed through the previous members of sequence (60):

$$
K_{M+1}=a_{M} K_{M}+a_{M-1} K_{M-1}+\cdots+a_{1} K_{1}+b_{1} P+b_{2} Q, \quad M \geq 1,
$$

where the operators $Q, P, K_{1}, \ldots K_{M}$ are linearly independent, the coefficients $a_{i}, b_{k}$ are functions of a finite set of dynamical variables $\bar{v}, \bar{v}_{x}, \bar{v}_{x x} \ldots$;

2) the operator $K_{1}$ is linearly expressed through $P, Q$ :

$$
K_{1}=b_{1} P+b_{2} Q .
$$

Let us begin with case 1). We commute both sides of (63) with the operator $D_{x}$ and apply formula (62). Comparing the coefficients at the independent operators and collecting the coefficients at the operator $K_{M}$ we get the equality

$$
D_{x}\left(a_{M}\right)=-\frac{1}{2}(M+1)(M+2) e^{v_{0}}
$$

which is never realized.

In case 2 ) by commuting both sides of (64) with the operator $D_{x}$ and by using formulae (61), (59) we get a contradictory equation:

$$
-e^{v_{0}}\left(v_{0}-v_{-1}-1\right) P-e^{v_{0}} Q=D_{x}\left(b_{1}\right) P+D_{x}\left(b_{2}\right) Q-b_{1} e^{v_{0}} X_{0}+b_{2} e^{v_{0}}\left(v_{0}-v_{-1}\right) X_{0} .
$$

Indeed, by comparing the coefficients at $Q$ we obtain the relation $D_{x}\left(b_{2}\right)=-e^{v_{0}}$ which has no solutions depending on a finite set of dynamical variables. Therefore this case is also not realised. In other words, equation (56) is not integrable in the sense of Definition 1.

Let us turn to equation (57) with $m \neq 1,2$. It is easily verified that operator $Z_{0,-1}=$ $\left[X_{-1},\left[X_{0}, Z\right]\right]$ satisfies the following commutativity relation:

$$
\begin{aligned}
& {\left[D_{x}, Z_{0,-1}\right]=\left(A e^{-v_{-1}+\kappa\left(v_{0}-v_{-2}\right)}+B e^{\mu v_{-1}+\kappa v_{-2}}\right) X_{-1}+} \\
& +\left(A e^{-v_{0}+\kappa\left(v_{1}-v_{-1}\right)}+B e^{\mu v_{0}+\kappa v_{-1}}\right) X_{0}+\left(A e^{-v_{1}+\kappa\left(v_{2}-v_{0}\right)}+B e^{\mu v_{1}+\kappa v_{0}}\right) X_{1},
\end{aligned}
$$


where $\mu=-1+\frac{m}{2}, \kappa=\frac{m}{2}, A=-(m-2) \frac{m}{2}, B=\frac{m(m-1)(m-2)}{2}$. Note that $A \neq 0, B \neq 0$. We define the polynomial $\sigma(\lambda)=(\lambda+1)(\lambda-\mu)(\lambda+\kappa)$ to obtain the formulae

$$
\begin{gathered}
{\left[D_{x}, \sigma\left(a d_{X_{-1}}\right) Z_{0,-1}\right]=B e^{\mu v_{0}} \sigma\left(X_{-1}\right) e^{\kappa v_{-1}} X_{0},} \\
{\left[D_{x}, \sigma\left(a d_{X_{1}}\right) Z_{0,-1}\right]=A e^{-\kappa v_{-1}-v_{0}} \sigma\left(X_{1}\right) e^{\kappa v_{1}} X_{0} .}
\end{gathered}
$$

Evaluation gives $\sigma\left(X_{-1}\right) e^{\kappa v_{-1}}=\frac{m(m+2)}{2} e^{\kappa v_{-1}}$ and $\sigma\left(X_{1}\right) e^{\kappa v_{1}}=\frac{m(m+2)}{2} e^{\kappa v_{1}}$. Let us introduce the notation $\sigma\left(a d_{X_{-1}}\right) Z_{0,-1}=P_{0}, \sigma\left(a d_{X_{1}}\right) Z_{0,-1}=P_{1}, B_{0}=B \frac{m(m+2)}{2}, A_{0}=A \frac{m(m+2)}{2}$, and rewrite the last two equations in the form

$$
\left[D_{x}, P_{0}\right]=B_{0} e^{\mu v_{0}+\kappa v_{-1}} X_{0}, \quad\left[D_{x}, P_{1}\right]=A_{0} e^{-v_{0}+\kappa v_{1}-\kappa v_{-1}} X_{0} .
$$

According to the results of [12] the Lie-Rinehart algebra generated by the operators $P_{0}, P_{1}$ is finite-dimensional only if $\mu=-1$, i.e., for $m=0$. So we obtain the system of independent equations of the form $v_{n, x y}=4 e^{-v_{n}}$ (each of these equations is the well-known integrable Liouville equation). This finishes the analysis of Subcases 1-2.

\subsection{Subcase 3}

Among dispersionless equations of the third subcase, only one gives rise to an integrable equation of type (1), namely, to the third case of Theorem 1:

$$
u_{x y}=u_{x} \triangle_{z \bar{z}} u \text {. }
$$

It is shown below that (65) can be derived from equations (16) and (17) under a suitable choice of the parameters. By using the discretization rule explained in Section 3 we convert equations (16) - (19) to the following ones:

$$
\begin{aligned}
& u_{n, x y}=-\frac{\beta^{\prime \prime}\left(u_{n}\right)}{\beta^{\prime}\left(u_{n}\right)} u_{n, x} u_{n, y}+\beta^{\prime}\left(u_{n}\right)\left(u_{n+1}-2 u_{n}+u_{n-1}\right) u_{n, y}+ \\
& +\left(\frac{1}{2} \alpha^{2} \beta\left(u_{n}\right)+\frac{3}{2} \alpha \beta^{\prime}\left(u_{n}\right) \sqrt{\left(u_{n+1}-u_{n}\right)\left(u_{n}-u_{n-1}\right)}+\beta^{\prime \prime}\left(u_{n}\right)\left(u_{n+1}-u_{n}\right)\left(u_{n}-u_{n-1}\right)\right) u_{n, y} \\
& u_{n, x y}=-\frac{\beta^{\prime}\left(u_{n}\right)}{\beta\left(u_{n}\right)} u_{n, x} u_{n, y}+ \\
& +\left(\gamma+\beta\left(u_{n}\right) u_{n, y}\right)\left(u_{n+1}-2 u_{n}+u_{n-1}+\frac{\delta}{\beta\left(u_{n}\right)}+\frac{\beta^{\prime}\left(u_{n}\right)}{\beta\left(u_{n}\right)}\left(u_{n+1}-u_{n}\right)\left(u_{n}-u_{n-1}\right)\right), \\
& u_{n, x y}=-\frac{\beta^{\prime \prime}\left(u_{n}\right)}{\beta^{\prime}\left(u_{n}\right)} u_{n, x} u_{n, y}+ \\
& \quad+\gamma e^{\frac{1}{2} \alpha \beta\left(u_{n}\right)+\beta^{\prime}\left(u_{n}\right) \sqrt{\left(u_{n+1}-u_{n}\right)\left(u_{n}-u_{n-1}\right)}}\left(\alpha+2 \beta^{\prime}\left(u_{n}\right)\left(u_{n+1}-2 u_{n}+u_{n-1}\right)+\right. \\
& \left.\quad+\alpha \beta^{\prime}\left(u_{n}\right) \sqrt{\left(u_{n+1}-u_{n}\right)\left(u_{n}-u_{n-1}\right)}+2 \beta^{\prime \prime}\left(u_{n}\right)\left(u_{n+1}-u_{n}\right)\left(u_{n}-u_{n-1}\right)\right) u_{n, y}, \quad
\end{aligned}
$$




$$
\begin{aligned}
u_{n, x y}= & -\frac{\beta^{\prime}\left(u_{n}\right)}{\beta\left(u_{n}\right)} u_{n, x} u_{n, y}+\delta e^{\beta\left(u_{n}\right) \sqrt{\left(u_{n+1}-u_{n}\right)\left(u_{n}-u_{n-1}\right)}} \times \\
& \times\left(\beta\left(u_{n}\right)\left(u_{n+1}-2 u_{n}+u_{n-1}\right)+\beta^{\prime}\left(u_{n}\right)\left(u_{n+1}-u_{n}\right)\left(u_{n}-u_{n-1}\right)\right)\left(u_{n, y}+\frac{\gamma}{\beta\left(u_{n}\right)}\right),
\end{aligned}
$$

respectively. These equations are of the form

$$
u_{n, x y}=s\left(u_{n}\right) u_{n, x} u_{n, y}+q\left(u_{n-1}, u_{n}, u_{n+1}\right) u_{n, y}
$$

where $s\left(u_{n}\right)=-\frac{\beta^{\prime \prime}\left(u_{n}\right)}{\beta^{\prime}\left(u_{n}\right)}$ for $(66),(68)$, and $s\left(u_{n}\right)=-\frac{\beta^{\prime}\left(u_{n}\right)}{\beta\left(u_{n}\right)}$ for $(67),(69)$. In the first case we apply the point transformation $v_{n}=\beta\left(u_{n}\right)$, that is, $u_{n}=\varphi\left(v_{n}\right), \varphi=\beta^{-1}$. In the second case we use $v_{n}=\psi\left(u_{n}\right)$ where $\psi^{\prime}=\beta$, that is, $u_{n}=\varphi\left(v_{n}\right), \varphi=\psi^{-1}$. Thus, equations (70) take the form

$$
v_{n, x y}=g\left(v_{n+1}, v_{n}, v_{n-1}\right) v_{n, y}+r\left(v_{n+1}, v_{n}, v_{n-1}\right)
$$

where $\frac{\partial g\left(v_{n+1}, v_{n}, v_{n-1}\right)}{\partial v_{n \pm 1}} \neq 0$. Namely,

$$
\begin{aligned}
& v_{n, x y}=\beta^{\prime 2}\left(u_{n}\right)\left(\varphi\left(v_{n+1}\right)-2 \varphi\left(v_{n}\right)+\varphi\left(v_{n-1}\right)\right) \varphi^{\prime}\left(v_{n}\right) v_{n, y}+ \\
& +\beta^{\prime}\left(u_{n}\right)\left(\frac{1}{2} \alpha^{2} \beta\left(u_{n}\right)+\frac{3}{2} \alpha \beta^{\prime}\left(u_{n}\right) \sqrt{\left(\varphi\left(v_{n+1}\right)-\varphi\left(v_{n}\right)\right)\left(\varphi\left(v_{n}\right)-\varphi\left(v_{n-1}\right)\right)}+\right. \\
& \left.+\beta^{\prime \prime}\left(u_{n}\right)\left(\varphi\left(v_{n+1}\right)-\varphi\left(v_{n}\right)\right)\left(\varphi\left(v_{n}\right)-\varphi\left(v_{n-1}\right)\right)\right) \varphi^{\prime}\left(v_{n}\right) v_{n, y}, \\
& v_{n, x y}=\beta\left(u_{n}\right)\left(\gamma+\beta\left(u_{n}\right) \varphi^{\prime}\left(v_{n}\right) v_{n, y}\right)\left(\varphi\left(v_{n+1}\right)-2 \varphi\left(v_{n}\right)+\varphi\left(v_{n-1}\right)+\frac{\delta}{\beta\left(u_{n}\right)}+\right. \\
& \left.+\frac{\beta^{\prime}\left(u_{n}\right)}{\beta\left(u_{n}\right)}\left(\varphi\left(v_{n+1}\right)-\varphi\left(v_{n}\right)\right)\left(\varphi\left(v_{n}\right)-\varphi\left(v_{n-1}\right)\right)\right), \\
& v_{n, x y}=\beta^{\prime}\left(u_{n}\right) \gamma e^{\frac{1}{2} \alpha \beta\left(u_{n}\right)+\beta^{\prime}\left(u_{n}\right) \sqrt{\left(\varphi\left(v_{n+1}\right)-\varphi\left(v_{n}\right)\right)\left(\varphi\left(v_{n}\right)-\varphi\left(v_{n-1}\right)\right)}} \times \\
& \times\left(\alpha+2 \beta^{\prime}\left(u_{n}\right)\left(\varphi\left(v_{n+1}\right)-2 \varphi\left(v_{n}\right)+\varphi\left(v_{n-1}\right)\right)+\alpha \beta^{\prime}\left(u_{n}\right) \sqrt{\left(\varphi\left(v_{n+1}\right)-\varphi\left(v_{n}\right)\right)\left(\varphi\left(v_{n}\right)-\varphi\left(v_{n-1}\right)\right)}+\right. \\
& \left.+2 \beta^{\prime \prime}\left(u_{n}\right)\left(\varphi\left(v_{n+1}\right)-\varphi\left(v_{n}\right)\right)\left(\varphi\left(v_{n}\right)-\varphi\left(v_{n-1}\right)\right)\right) \varphi^{\prime}\left(v_{n}\right) v_{n, y}, \\
& v_{n, x y}=\beta\left(u_{n}\right) \delta e^{\beta\left(u_{n}\right) \sqrt{\left(\varphi\left(v_{n+1}\right)-\varphi\left(v_{n}\right)\right)\left(\varphi\left(v_{n}\right)-\varphi\left(v_{n-1}\right)\right)}} \times \\
& \times\left(\beta\left(u_{n}\right)\left(\varphi\left(v_{n+1}\right)-2 \varphi\left(v_{n}\right)+\varphi\left(v_{n-1}\right)\right)+\beta^{\prime}\left(u_{n}\right)\left(\varphi\left(v_{n+1}\right)-\varphi\left(v_{n}\right)\right)\left(\varphi\left(v_{n}\right)-\varphi\left(v_{n-1}\right)\right)\right) \times \\
& \times\left(\varphi^{\prime}\left(v_{n}\right) v_{n, y}+\frac{\gamma}{\beta\left(u_{n}\right)}\right) .
\end{aligned}
$$

Thus, we can apply Proposition 2. A simple analysis shows that equations (73), (74) are never reduced to $(34),(35)$. Let us calculate mixed partial derivatives of the right-hand sides $\tilde{f}, \tilde{\tilde{f}}$ of (71), (72), respectively:

$$
\begin{gathered}
\tilde{f}_{v_{n+1}, v_{n-1}}=-\beta^{\prime}\left(u_{n}\right) \beta^{\prime \prime}\left(u_{n}\right) \varphi^{\prime}\left(v_{n+1}\right) \varphi^{\prime}\left(v_{n-1}\right) \varphi^{\prime}\left(v_{n}\right) v_{n, y} \\
\tilde{\tilde{f}}_{v_{n+1}, v_{n-1}}=-\beta\left(u_{n}\right) \beta^{\prime}\left(u_{n}\right) \varphi^{\prime}\left(v_{n+1}\right) \varphi^{\prime}\left(v_{n-1}\right) \varphi^{\prime}\left(v_{n}\right) v_{n, y} .
\end{gathered}
$$


Due to formulae (34), (35) the equalities $\tilde{f}_{v_{n+1}, v_{n-1}}=0, \tilde{\tilde{f}}_{v_{n+1}, v_{n-1}}=0$ should be satisfied. Consequently, we have $\beta^{\prime \prime}=0$ in case (75) and $\beta^{\prime}=0$ in case (76). Thus, equations (66), (67) take the form

$$
\begin{gathered}
u_{n, x y}=c_{1}\left(u_{n+1}-2 u_{n}+u_{n-1}\right) u_{n, y}, \\
u_{n, x y}=\left(\gamma+c_{1} u_{n, y}\right)\left(u_{n+1}-2 u_{n}+u_{n-1}+\frac{\delta}{c_{1} u+c_{2}}\right) .
\end{gathered}
$$

Due to Proposition 2 both of these equations have to concide with equation (35). Thus, we conclude that $\delta=0$ in (78). Then (78) is reduced to (35) by the changes of variables $u_{n} \rightarrow$ $u_{n}-\frac{\gamma}{c_{1}} y, x \rightarrow \frac{x}{c_{1}}$. Equation (77) is reduced to (35) by the change of variables $x \rightarrow \frac{x}{c_{1}}$. Ultimately, both of them are equivalent to the third case of Theorem 1.

\subsection{Subcase 4}

It is readily seen that equations of Subcase 4 give rise to lattice equations of type (29). For this class the complete classification is given in [10] (see Proposition 1 above). Hence investigation of the equations (20)-(25) is straightforward. This will result in the last three cases of Theorem 1. Let us first concentrate on the equations (20)-(22), which are integrable for a certain choice of parameters. They correspond to lattice equations of the form:

$$
\begin{aligned}
& u_{n, x y}=\frac{2\left(u_{n+1}-2 u_{n}+u_{n-1}\right)+\left(4 \beta^{\prime}\left(u_{n}\right)-\alpha\right) \sqrt{\left(u_{n+1}-u_{n}\right)\left(u_{n}-u_{n-1}\right)}+2 \beta \beta^{\prime}-\alpha \beta}{2\left(\sqrt{\left(u_{n+1}-u_{n}\right)\left(u_{n}-u_{n-1}\right)}+\beta\left(u_{n}\right)\right)^{2}} u_{n, x} u_{n, y}, \\
& u_{n, x y}=\frac{u_{n, x} u_{n, y}+\beta\left(u_{n}\right) u_{n, x}}{\left(\sqrt{\left(u_{n+1}-u_{n}\right)\left(u_{n}-u_{n-1}\right)}+\gamma \beta\left(u_{n}\right)\right)^{2}}\left(u_{n+1}-2 u_{n}+u_{n-1}\right)+ \\
& +\frac{\left(4 \gamma \beta^{\prime}\left(u_{n}\right)-\alpha\right) \sqrt{\left(u_{n+1}-u_{n}\right)\left(u_{n}-u_{n-1}\right)}+2 \gamma^{2} \beta\left(u_{n}\right) \beta^{\prime}\left(u_{n}\right)-\alpha \gamma \beta\left(u_{n}\right)}{2\left(\sqrt{\left(u_{n+1}-u_{n}\right)\left(u_{n}-u_{n-1}\right)}+\gamma \beta\left(u_{n}\right)\right)^{2}} u_{n, x} u_{n, y}- \\
& -\frac{2 \beta^{\prime}\left(u_{n}\right)\left(u_{n+1}-u_{n}\right)\left(u_{n}-u_{n-1}\right)+\alpha \beta\left(u_{n}\right) \sqrt{\left(u_{n+1}-u_{n}\right)\left(u_{n}-u_{n-1}\right)}+\alpha \gamma \beta^{2}\left(u_{n}\right)}{2\left(\sqrt{\left(u_{n+1}-u_{n}\right)\left(u_{n}-u_{n-1}\right)}+\gamma \beta\left(u_{n}\right)\right)^{2}} u_{n, x}, \\
& u_{n, x y}=\frac{\left(u_{x}+\beta\left(u_{n}\right)\right)\left(u_{y}+\delta \beta\left(u_{n}\right)\right)}{\left(\sqrt{\left(u_{n+1}-u_{n}\right)\left(u_{n}-u_{n-1}\right)}+\gamma \beta\left(u_{n}\right)\right)^{2}}\left(u_{n+1}-2 u_{n}+u_{n-1}\right)+ \\
& +\frac{\left(4 \gamma \beta^{\prime}\left(u_{n}\right)-\alpha\right) \sqrt{\left(u_{n+1}-u_{n}\right)\left(u_{n}-u_{n-1}\right)}+2 \gamma^{2} \beta\left(u_{n}\right) \beta^{\prime}\left(u_{n}\right)-\alpha \gamma \beta\left(u_{n}\right)}{2\left(\sqrt{\left(u_{n+1}-u_{n}\right)\left(u_{n}-u_{n-1}\right)}+\gamma \beta\left(u_{n}\right)\right)^{2}} u_{n, x} u_{n, y}- \\
& -\frac{2 \beta^{\prime}\left(u_{n}\right)\left(u_{n+1}-u_{n}\right)\left(u_{n}-u_{n-1}\right)+\alpha \beta\left(u_{n}\right) \sqrt{\left(u_{n+1}-u_{n}\right)\left(u_{n}-u_{n-1}\right)}+\alpha \gamma \beta^{2}\left(u_{n}\right)}{2\left(\sqrt{\left(u_{n+1}-u_{n}\right)\left(u_{n}-u_{n-1}\right)}+\gamma \beta\left(u_{n}\right)\right)^{2}} \times \\
& \times\left(u_{y}+\delta u_{x}+\delta \beta\left(u_{n}\right)\right) .
\end{aligned}
$$


More precisely, equations (79), (80) are integrable only when $\alpha=\beta=0$, and then they coincide with (30). Equation (81) is more generic, its integrable cases are obtained by setting:

$$
\begin{aligned}
& \text { i) } \alpha=0, \beta=0 \\
& \text { ii) } \alpha=0, \beta=0, \gamma=0, \delta=0 \\
& \text { iii) } \alpha=0, \gamma=0, \delta \neq 0, \beta=-u_{n} \\
& \text { iv) } \alpha=0, \gamma=0, \delta \neq 0, \beta=-u_{n}^{2}-1 .
\end{aligned}
$$

In the first two cases we get equation (30); for $i i i)$ and $i v$ ) equation (81) is reduced to (31) and, respectively, to (32). As for equations (23)-(25), they are not integrable since they don't satisfy the integrability conditions derived in [10].

Modulo elementary equivalence transformations, the final list of lattice equations (1) passing both tests is as follows:

1) $u_{n, x y}=e^{u_{n+1}-2 u_{n}+u_{n-1}}$,

2) $u_{n, x y}=e^{u_{n+1}}-2 e^{u_{n}}+e^{u_{n-1}}$,

3) $u_{n, x y}=\left(u_{n+1}-2 u_{n}+u_{n-1}\right) u_{n, x}$,

4) $u_{n, x y}=\alpha_{n} u_{n, x} u_{n, y}, \quad \alpha_{n}=\frac{1}{u_{n}-u_{n-1}}-\frac{1}{u_{n+1}-u_{n}}=\frac{u_{n+1}-2 u_{n}+u_{n-1}}{\left(u_{n+1}-u_{n}\right)\left(u_{n}-u_{n-1}\right)}$,

5) $u_{n, x y}=\alpha_{n}\left(u_{n, x}-u_{n}\right)\left(u_{n, y}-u_{n}\right)+u_{n, x}+u_{n, y}-u_{n}$,

6) $u_{n, x y}=\alpha_{n}\left(u_{n, x}-u_{n}^{2}-1\right)\left(u_{n, y}-u_{n}^{2}-1\right)+2 u_{n}\left(u_{n, x}+u_{n, y}-u_{n}^{2}-1\right)$.

All of them are equivalent to the normal forms of Theorem 1.

Remark. Note that equation 2, which is not exactly of the form (1), can be transformed into this form: rewriting it as $u_{x y}=\triangle_{z \bar{z}} e^{u}$ and setting $u=\ln v$ we obtain

$$
v_{x y}=\frac{v_{x} v_{y}}{v}+v \triangle_{z \bar{z}} v
$$

On the other hand, the integrable equation $u_{n, x y}=e^{u_{n+1}-u_{n}}-e^{u_{n}-u_{n-1}}$, which is in fact equivalent to equation 2 , is formally not of type (1) and therefore does not appear on the list. Integrable lattice (34) is also absent from the list for the same reason.

\section{Acknowledgements}

We thank Maxim Pavlov for useful discussions. The research of EVF was supported by the EPSRC grant EP/N031369/1.

Data Availability Statement. The data (Mathematica programme of symbolic computations performed in Section 2) that support the findings of this paper are available from the corresponding author upon request. 


\section{References}

[1] D.M.J. Calderbank, Integrable background geometries, SIGMA 10 (2014) 51 pp.

[2] D.M.J. Calderbank, B. Kruglikov, Integrability via geometry: dispersionless differential equations in three and four dimensions, to appear in Proc. Roy. Soc. London Ser. A; arXiv:1612.02753.

[3] E. Cartan, Sur une classe d'espaces de Weyl, Ann. Sci. École Norm. Sup. (3) 60 (1943) $1-16$.

[4] E. Cartan, The geometry of differential equations of third order, Revista Mat. Hisp.-Amer. 4 (1941) 3-33.

[5] M. Dunajski, L.J. Mason and P. Tod, Einstein-Weyl geometry, the dKP equation and twistor theory, J. Geom. Phys. 37, no. 1-2 (2001) 63-93.

[6] M. Dunajski, A class of Einstein-Weyl spaces associated to an integrable system of hydrodynamic type, J. Geom. Phys. 51, no. 1 (2004) 126-137.

[7] M. Dunajski, E.V. Ferapontov, B. Kruglikov, On the Einstein-Weyl and conformal selfduality equations, J. Math. Phys. 56, 083501 (2015); doi: 10.1063/1.4927251.

[8] E.V. Ferapontov, Laplace transforms of hydrodynamic-type systems in Riemann invariants, Theoret. and Math. Phys. 110 (1997) 68-77.

[9] E.V. Ferapontov, B.S. Kruglikov, Dispersionless integrable systems in 3D and Einstein-Weyl geometry, J. Diff. Geom. 97 (2014) 215-254.

[10] I. T. Habibullin, M. N. Kuznetsova, A classification algorithm for integrable twodimensional lattices via Lie-Rinehart algebras, Theoret. and Math. Phys., 203, no. 1 (2020) 569-581.

[11] I. Habibullin, M. Poptsova, Classification of a subclass of two-dimensional lattices via characteristic Lie rings, SIGMA 13, no. 073 (2017) 26 pp.

[12] I.T. Habibullin, M.N. Kuznetsova, A.U. Sakieva, Integrability conditions for twodimensional lattices, arXiv:2005.09712.

[13] N.J. Hitchin, Complex manifolds and Einstein's equations, Twistor geometry and nonlinear systems (Primorsko, 1980), 73-99, Lecture Notes in Math. 970, Springer, Berlin-New York (1982).

[14] P.E. Jones and K.P. Tod, Minitwistor spaces and Einstein-Weyl spaces, Classical Quantum Gravity 2, no. 4 (1985) 565-577.

[15] M.N. Kuznetsova, Classification of a subclass of quasilinear two-dimensional lattices by means of characteristic algebras, Ufa Math. J. 11, no. 3 (2019) 109-131. 
[16] D. Millionshchikov, Lie algebras of slow growth and Klein-Gordon PDE, Algebr. Represent. Theor. https://doi.org/10.1007/s10468-018-9794-4 (2018).

[17] G. Rinehart, Differential forms for general commutative algebras, Trans. Amer. Math. Soc. 108 (1963) 195-222.

[18] A.B. Shabat, R.I. Yamilov, To a transformation theory of two-dimensional integrable systems, Phys. Lett. A 227 (1997) 15-23.

[19] A.B. Shabat, R.I. Yamilov, Exponential systems of type I and the Cartan matrix, Preprint, Bashkir branch of AS USSR, Ufa, (1981) 22pp.

[20] R.S. Ward, Einstein-Weyl spaces and $S U(\infty)$ Toda fields, Class. Quantum Grav. 7, no. 4 (1990) L95-L98.

[21] V.E. Zakharov, Dispersionless limit of integrable systems in $2+1$ dimensions, in Singular Limits of Dispersive Waves, Ed. N.M. Ercolani et al., Plenum Press, NY (1994) 165-174.

[22] A.V. Zhiber, R.D. Murtazina, I.T. Habibullin, A.B. Shabat, Characteristic Lie rings and nonlinear integrable equations, M.-Izhevsk: Institute of Computer Science, (2012) 376 pp.

[23] A.V. Zhiber, O.S. Kostrigina, Exactly integrable models of wave processes, Vestnik USATU, 9:7(25) (2007) 83-89. 